\title{
THE GLOBAL MCKAY-RUAN CORRESPONDENCE VIA MOTIVIC INTEGRATION
}

\author{
ERNESTO LUPERCIO AND MAINAK PODDAR
}

\begin{abstract}
The purpose of this paper is to show how the methods of motivic integration of Kontsevich, Denef-Loeser and Looijenga can be adapted to prove the McKay-Ruan correspondence, a generalization of the McKay-Reid correspondence to orbifolds that are not necessarily global quotients.
\end{abstract}

\section{INTRODUCTION}

1.1. In this paper when we say orbifold we mean an algebraic variety with quotient singularities. We will always work over the field $\mathbb{C}$. We say that an orbifold $X$ is a global quotient if it is of the form $X=U / G$ for $U$ smooth and $G$ a finite group. There are simple examples of orbifolds that are not global quotients such as weighted projective spaces like, for example, $W P(1,1,2)$. An $n$-dimensional orbifold is Gorenstein or $S L$ if all the local isotropy groups are finite subgroups of $\mathrm{SL}_{n}(\mathbb{C})$. Given two Gorenstein or $S L$ orbifolds $X$ and $Y$ we say that they are $K$-equivalent if there is a common birational resolution $\phi: Z \rightarrow X, \psi: Z \rightarrow Y$ so that $\phi^{*} K_{X}=\psi^{*} K_{Y}$.

1.2. The cohomological McKay-Reid correspondence states that for an $S L$ global quotient orbifold having a smooth crepant resolution, there is a certain correspondence between the cohomology generators of the resolution and the conjugacy classes of the group $G$. A very good introduction to this subject, including its relation to the classical McKay correspondence can be found in 20. We refer the reader to [3, 7, 10, 11] for detailed results. Yongbin Ruan (cf. 21] Section 6.4) conjectured a generalization of this correspondence to general orbifolds, which has the added advantage that the smoothness of the resolution is no longer essential. In the McKay-Ruan correspondence (stated below as Theorem 1.4.1), the role of the conjugacy classes is taken over by the twisted sectors of the orbifold in question (see [4, 8, 12] and Definition 3.3.1 below).

1.3. The purpose of this note is to show how the methods of motivic integration of Kontsevich [13, Denef-Loeser 6, 7] and Looijenga [14 can be adapted to prove the McKay-Ruan correspondence, namely the following theorem.

Theorem 1.3.1. If the orbifolds $X$ and $Y$ are $K$-equivalent and complete then their orbifold Hodge numbers, orbifold Hodge structures and orbifold Euler characteristics coincide.

This theorem has been proved independently by T. Yasuda 23] as we were informed by an email from him during the preparation of this note.

2000 Mathematics Subject Classification. 14A20, 14E15, 14F43. 
1.4. The previous theorem is actually a consequence of the transformation rule for the motivic measure (Theorem 1.16 in [7, Theorem 3.2 in [14), and the following formula that is analogous to Theorem 8.1 in [14] except that in there the orbifold was only a global quotient.

Theorem 1.4.1.

$$
\mu^{\mathrm{orb}}(\mathcal{L}(X))=\sum_{\alpha \in \pi_{0}(\wedge X)}\left[X^{\alpha} / X\right] \mathbb{L}^{w(\alpha)} \in \hat{M}_{X}\left[\mathbb{L}^{1 / m}\right]
$$

Let us briefly describe the different terms involved in this formula (cf. [14]). $\mathcal{L}(X)$ is the space of $\operatorname{arcs}$ in $X$ whose $\mathbb{C}$-points correspond to formal arcs $\mathbb{D} \rightarrow X$. Here $\mathbb{D}=\operatorname{Spec} \mathbb{C}[[z]]$. The motivic ring $M_{X}$ is equal to the localization of $K_{0}\left(\mathcal{V}_{X}\right)$, the Grothendieck ring of $X$-varieties (plus the relation 1.10 in 7 ]), with respect to the Tate motive $\mathbb{L}=\left[\mathbb{A}^{1}\right] \in K_{0}\left(\mathcal{V}_{X}\right)$. The $M_{X}\left[\mathbb{L}^{1 / m}\right]$ valued measure $\mu^{\text {orb }}$ on $\mathcal{L}(X)$ is defined by Denef-Loeser in [7] $\S 2.7$ (cf. [14] $\S 8$ ). This measure is determined by the dualizing sheaf of $X$ if $X$ is Gorenstein. We write $\wedge X$ to denote the twisted sectors or inertia orbifold of $X$ (see Definition 3.3.1 cf. 16 definition 3.6.5.). Finally the numbers $w(\alpha)$ are the degree shifting numbers described by Chen-Ruan [4. They are also known as Fermionic degree shifting numbers in the physics literature and sometimes referred to as age in the terminology of Miles Reid.

\section{The Case of the Global Quotient.}

2.1. The proof of Theorem 1.4.1 in the global quotient case can be found in [7, 14. The proof in the general case follows closely the proof of the McKay-Reid correspondence (Theorem 8.1 in [14, cf. 2, 7]) described by Looijenga 14, Section 8. We briefly recall the basic idea of this argument now (cf. 20] formula 4.5). We divide the proof into two steps.

2.2. Step I. Let $p: U \rightarrow X$ be the quotient map associated to $X$. We set $\mathcal{L}^{\circ}(X)$ to be the set of arcs in $X$ not contained in the discriminant of $p$ (this is written $\mathcal{L}^{\prime}(X)$ in 14 and $\mathcal{L}^{g}(X)$ in $[7)$.

We denote by $[m]: \mathbb{D} \rightarrow \mathbb{D}$ the $m$-th power map $z^{1 / m} \mapsto z$. Let $\zeta_{m}=\exp (2 \pi i / m)$ be a primitive $m$-root of unity where $m$ is the order of $G$.

The first thing to do is to verify the following decomposition (cf. [20] (4.6), [7] 6.2 and [16] $\S 6.1 .2)$

$$
\mathcal{L}^{\circ}(X)=\coprod_{(g) \in \operatorname{Conj}(G)} \mathcal{L}_{g}^{\circ}(X) .
$$

Here $\mathcal{L}_{g}^{\circ}(X)$ consists of the $\operatorname{arcs} \gamma$ in $\mathcal{L}^{\circ}(X)$ that have a lift $\tilde{\gamma}$ in $\mathcal{L}^{\circ}(U)$ with the following property

$$
g \tilde{\gamma}=\gamma \zeta_{m}
$$

We call $\mathcal{L}_{g}^{\circ}(X)$ the $\operatorname{arcs}$ on $X$ twisted by $g$.

2.3. Step II. The next step is to verify the following identity,

$$
\mu^{\text {orb }}\left(\mathcal{L}_{g}(X)\right)=\left[X^{g} / X\right] \mathbb{L}^{w\left(g^{-1}\right)}
$$

where $X^{g}=U^{g} / C(g)$ is the $(g)$-twisted sector, namely the fixed points of $g$ modulo its centralizer.

This calculation is performed using the so-called change of variables formula (see 7] Theorem 1.16, [5] Theorem 2.18, 14] Theorem 3.2) for the motivic integral defining the orbifold motivic measure. This is done for example in [7] $\S 3$. 
2.4. Finally all there is to do is to add over all $(g) \in \operatorname{Conj}(G)$ to get

$$
\mu^{\text {orb }}(\mathcal{L}(X))=\mu^{\text {orb }}\left(\mathcal{L}^{\circ}(X)\right)=\sum_{(g)}\left[X^{g} / X\right] \mathbb{L}^{w\left(g^{-1}\right)} .
$$

Since $\pi_{0}(\wedge[U / G])=\operatorname{Conj}(G)$ (see [16] Proposition 6.2.1) we have Theorem 1.4.1] for the case $X=U / G$.

\section{The General Case.}

3.1. We can think of a general orbifold $X$ as a Deligne-Mumford stack and such a stack admits an atlas given by an étale separated groupoid $\mathrm{G}$ in schemes. The category of orbifolds is then equivalent to the category of groupoids up to Morita equivalence. We refer the reader to [18, 19, 1, 17, 16, 22, for details. In any case we will denote by $U=\mathrm{G}_{0}$ and $R=\mathrm{G}_{1}$ the smooth schemes of objects and morphisms (arrows) of the groupoid $\mathrm{G}$ respectively, and the structure maps by:

$$
\mathrm{G}_{1}{ }_{t}{ }_{s} \mathrm{G}_{1} \stackrel{m}{\longrightarrow} \mathrm{G}_{1} \stackrel{i}{\longrightarrow} \mathrm{G}_{1} \stackrel{s}{\stackrel{\mathrm{t}}{\longrightarrow}} \mathrm{G}_{0} \stackrel{e}{\longrightarrow} \mathrm{G}_{1}
$$

where $s$ and $t$ are the source and the target maps of morphisms, $m$ is the composition of two of them whenever the target of the first equals the source of the second, $i$ gives us the inverse morphism and $e$ assigns the identity arrow to every object. We will write $\mathcal{X}=[U / \mathrm{G}]$ for the associated stack and $X=U / \mathrm{G}$ for the corresponding coarse moduli space. As before we write $p: U \rightarrow X$ to denote the quotient morphism.

3.2. The Arc Groupoid. The following definitions are borrowed from 16. Let $\mathbb{D}_{k}=\operatorname{Spec} \mathbb{C}[z] / z^{k+1}$. We fix once and for all a positive integer $m$ so that the orders of all the stabilizers of $X$ divide $m$ (here we are assuming that $X$ is complete). As before consider the $m$-th power morphism $[m]: \mathbb{D}_{k} \rightarrow \mathbb{D}_{k}$ given by $z^{1 / m} \mapsto z$ and $\zeta_{m}=\exp (2 \pi i / m)$. We denote by $C_{m}$ the cyclic group generated by $\zeta_{m}$. Let $\mathrm{D}_{k}$ be the groupoid with $\left(\mathrm{D}_{k}\right)_{0}=\mathbb{D}_{k},\left(\mathrm{D}_{k}\right)_{1}=\mathbb{D}_{k} \times C_{m}, s=i d$ and $t$ being the Galois action of $\zeta_{m}$ in $\mathbb{D}_{k}$.

3.2.1. We define a $k$-jet on the groupoid $\mathrm{G}$ to be a morphism of groupoids

$$
\gamma: \mathrm{D}_{k} \rightarrow \mathrm{G} \text {. }
$$

We write $\gamma_{i}:\left(D_{k}\right)_{i} \rightarrow G_{i}$ for $i=0,1$ for the corresponding morphisms in objects and arrows of $\mathrm{D}_{k}$.

3.2.2. We define the $k$-jet groupoid $\mathrm{L}_{k} \mathrm{G}$ associated to $\mathrm{G}$ by the following data:

(i) Objects $\left(\left(\mathrm{L}_{k} \mathrm{G}\right)_{0}\right)$ : Morphisms $\mathrm{D}_{k} \rightarrow \mathrm{G}$.

(ii) Morphisms $\left(\left(\mathrm{L}_{k} \mathrm{G}\right)_{1}\right)$ : For two elements in $\left(\mathrm{L}_{k} \mathrm{G}\right)_{0}$, say $\Psi, \Phi: \mathrm{D}_{k} \rightarrow \mathrm{G}$ a morphism (arrow) from $\Psi$ to $\Phi$ is a morphism $\Lambda: \mathbb{D}_{k} \times C_{m} \rightarrow \mathrm{G}_{1}$ that makes the following diagram commute

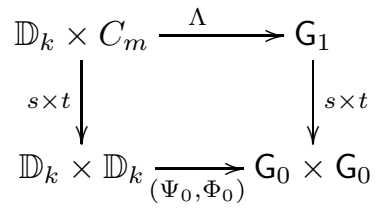

and such that for $r \in \mathbb{D}_{k} \times C_{m}$

$$
\Lambda(r)=\Psi_{1}(r) \cdot \Lambda(e s(r))=\Lambda(e t(r)) \cdot \Phi_{1}(r) .
$$


The composition of morphisms is defined pointwise, in other words, for $\Lambda$ and $\Omega$ with

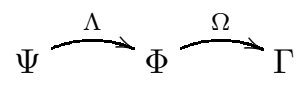

we set

$$
\Omega \circ \Lambda(e s(r)):=\Lambda(e s(r)) \cdot \Omega(e s(r))
$$

and

$$
\Omega \circ \Lambda(r):=\Omega \circ \Lambda(e s(r)) \cdot \Gamma(r)=\Psi(r) \cdot \Omega \circ \Lambda(e t(r)) .
$$

3.2.3. The scheme structure on the space of objects $\left(\mathrm{L}_{k} \mathrm{G}\right)_{0}$ is given by identifying it as a subscheme of $\mathcal{L}_{k}\left(\mathrm{G}_{1}\right)$, for $\gamma_{1}$ completely determines $\gamma$. Similarly $\left(\mathrm{L}_{k} \mathrm{G}\right)_{1}$ is naturally a subscheme of $\mathcal{L}_{k}\left(\mathrm{G}_{1}\right)^{m}$. In fact we can do better. By 3.2.4 in [16] or simply by recalling that $\mathrm{G}$ is étale we can show as in 3.2.6 [16 that $\mathrm{L}_{k} \mathrm{G}$ is actually an étale groupoid in schemes, but we will not need this.

3.2.4. The arc groupoid is similarly defined. We denote it by $\mathrm{LG}=\mathrm{L}_{\infty} \mathrm{G}$. Just as in [14] for $m \geq n$ we have projections $\pi_{n}^{m}: \mathrm{L}_{m} \mathrm{G} \rightarrow \mathrm{L}_{n} \mathrm{G}$. We will simply write $\pi_{n}$ for $\pi_{n}^{\infty}$.

3.3. Step I. Now we generalize 2.2 to the general case.

Definition 3.3.1. The inertia groupoid $\wedge \mathrm{G}$ is defined in the following way:

(i) Objects $(\wedge \mathrm{G})_{0}$ : Elements $v \in \mathrm{G}_{1}$ such that $s(v)=t(v)$.

(ii) Morphisms $(\wedge \mathrm{G})_{1}$ : For $v, w \in(\wedge \mathrm{G})_{0}$ an arrow $v \stackrel{\alpha}{\rightarrow} w$ is an element $\alpha \in \mathrm{G}_{1}$ such that $v \cdot \alpha=\alpha \cdot w$

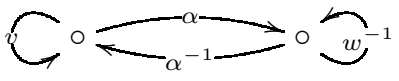

It is known that the inertia groupoid in the case of an orbifold matches with what is commonly known in the literature as twisted sectors [16].

The inertia groupoid defines a stack because as Moerdijk points out 18 it can be seen as

$$
\wedge \mathrm{G}=S_{\mathrm{G}} \rtimes \mathrm{G}
$$

where $S_{\mathrm{G}}=\Delta^{*}\left(\mathrm{G}_{1}\right)$ and $\Delta: \mathrm{G}_{0} \rightarrow \mathrm{G}_{0} \times \mathrm{G}_{0}$ is the diagonal morphism.

3.3.1. Notice that it is clear from the definitions that $\mathrm{L}_{0}(\mathrm{G})=\operatorname{Hom}(\overline{\mathbb{Z}}, \mathrm{G})=\wedge \mathrm{G}$ (cf. 3.6.4[16].) We will also call the map $\pi_{0}$ the evaluation map ev: $\mathrm{LG} \rightarrow \wedge$ G. We will write $\tau$ to denote the composition

$$
\tau: \mathrm{LG} \rightarrow \wedge \mathrm{G} \rightarrow \pi_{0}(\wedge \mathrm{G})
$$

and will write for $\alpha \in \pi_{0}(\wedge \mathrm{G})$

$$
\mathrm{L}_{\alpha}(\mathrm{G})=\tau^{-1}(\alpha)
$$

3.3.2. It may be worth pointing out here for the sake of comparison with the global quotient case that when $X=U / G$ we have $(6.2 .2$ [16] $)$

$$
\operatorname{Conj}(G)=\pi_{0}(\wedge(X))
$$

3.3.3. Let $p: U \rightarrow X$ be the quotient map associated to $X$. We set $\mathcal{L}^{\circ}(X)$ to be the set of $\operatorname{arcs}$ in $X$ not contained in the discriminant of $p$. (This is written $\mathcal{L}^{\prime}(X)$ in [14] and $\mathcal{L}^{g}(X)$ in [7].) Just as before the measure of $\mathcal{L}(X)-\mathcal{L}^{\circ}(X)$ is zero. (This is a local statement after all, but in any case it follows just as in 2.1 [7].) Compare with section 8 in [14. 
3.3.4. We write $\mathcal{L}_{\alpha}(U)$ to denote the arcs in $U$ of the form $\gamma_{1}$ for $\gamma$ an object in the jet groupoid lying in $\tau^{-1}(\alpha)$ (in the global quotient case this is what we called $\mathcal{L}_{g}(U)$.) Given an arc in $\mathcal{L}^{\circ}(X)$ the map $\gamma \circ[m]$ (where $[m]$ is defined in 3.2) lifts to a morphism $\mathrm{D} \rightarrow \mathrm{G}$. Let $\mathcal{L}_{\alpha}^{\circ}(X)$ the set of arcs corresponding to $\mathrm{L}_{\alpha} \mathrm{G}$ under this lift (cf. [14 discussion after 8.3).

3.3.5. We have the following decomposition

$$
\mathcal{L}^{\circ}(X)=\coprod_{\alpha \in \pi_{0}(\wedge \mathrm{G})} \mathcal{L}_{\alpha}^{\circ}(X) .
$$

This is true by $\S 2.1$ [7], for again this is a local statement.

3.4. Step II. We want to compute $\mu^{\text {orb }}\left(\mathcal{L}_{\alpha}^{\circ}(X)\right)$.

3.4.1. We will need the following stratification of an orbifold due to Haefliger 9 ] Proposition A.2.2.

Lemma 3.4.1. For an orbifold $X$ that is complete, there exist a stratification $X=\coprod_{i=1}^{N} X_{i}$ and a corresponding stratification of the étale groupoid $\mathrm{G}=\coprod_{i} \mathrm{G}_{i}$ so that each $X_{i}$ is smooth with stabilizer $\Gamma_{i}$ (where $\Gamma_{i}$ is a finite group) and each $\mathrm{G}_{i}$ is Morita equivalent to the action groupoid $X_{i} \times \Gamma_{i} \rightrightarrows X_{i}$.

Proof. By the results of [22] and [15] it is enough to consider the case when $X=$ $M / \mathrm{GL}_{n}(\mathbb{C})$ is given by the action groupoid $M \times \mathrm{GL}_{n}(\mathbb{C}) \rightrightarrows M$. Make a list of all possible stabilizers $G_{i}^{k}$ for $i=1, \ldots, N$, so that for a fixed $i_{0}$ all the $G_{i_{0}}^{k}$ are conjugate in $\mathrm{GL}_{n}(\mathbb{C})$. Let $N_{i}^{k}=N_{\mathrm{GL}_{n}(\mathbb{C})}\left(G_{i}^{k}\right)$ be the normalizer of $G_{i}^{k}, H_{i}^{k}=N_{i}^{k} / G_{i}^{k}$, and set $\Gamma_{i}=G_{i}^{k_{i}}$ for a fixed $k_{i}$. Let $M_{i}^{k}$ the subset of $M$ with stabilizer equal to $G_{i}^{k}$ (which is clearly constructible) and $M_{i}=\coprod_{k} M_{i}^{k}$. The groupoid $M_{i} \times G \rightrightarrows M_{i}$ is a subgroupoid of $M \times G \rightrightarrows M$. Note that $M_{i} \times G \rightrightarrows M_{i}$ defines the same orbifold as $M_{i}^{k_{i}} \times N_{i}^{k_{i}} \rightrightarrows M_{i}^{k_{i}}$. Set $X_{i}=M_{i}^{k_{i}} / H_{i}^{k_{i}}$ (which is smooth because $H_{i}^{k}$ acts freely on $M_{i}^{k}$ ), then the groupoid $M_{i}^{k_{i}} \times N_{i}^{k_{i}} \rightrightarrows M_{i}^{k_{i}}$ defines the same orbifold as $X_{i} \times \Gamma_{i} \rightrightarrows X_{i}$.

3.4.2. We will restrict our attention to a fixed stratum $X_{i}$ of $X$ and consider the arcs based at $X_{i}$ that we will write $\mathcal{L}(X)_{X_{i}}$ (cf. $\S 2.1[7]$ ). We will follow DenefLoeser $\S 3$ [7] to compute $\mu^{\text {orb }}\left(\mathcal{L}_{\alpha}^{\circ}(X)_{X_{i}}\right)$, and obtain the desired result by summing over strata.

3.4.3. Write $\mathrm{G}_{i}=\left[R_{i} \rightrightarrows U_{i}\right] \simeq\left[X_{i} \times \Gamma_{i} \rightrightarrows X_{i}\right]$ to denote the étale groupoid corresponding to $X_{i}$ and $p_{i}: U_{i} \rightarrow X_{i}$ the corresponding quotient map. Let $\nu_{i}$ denote the normal bundle of $U_{i}$ in $U$.

3.4.4. Since $\mathcal{L}_{\alpha}^{\circ}(X)_{X_{i}}$ depends only on a formal neighborhood $V_{i}$ of $X_{i}$ in $X$ and by decomposing the normal bundle $\nu_{i}=\oplus \nu_{i, \alpha}^{k}$ (cf. Lemma 8.4 [14], Lemma 2.2 [7]) on eigenspaces for a generator $g_{\alpha}$ of $\alpha$ in $\Gamma_{i}$ (the group $\Gamma_{i}$ acts trivially on $U_{i}$ and therefore linearly by fibers on $\nu_{i}$ ) we can define $\tilde{\lambda}: \nu_{\mathbb{C}[z]} \rightarrow X \otimes \mathbb{C}[z]$ by the formula 2.3[7] used fiberwise. We define $w(\alpha)=\sum_{k}(1-k / m) \operatorname{rank}\left(\nu_{i, \alpha}^{k}\right)$ (as in 8.4 [14]). This number is independent of $i$ (cf. 4]). By $2.3[7]$ we have that there is a $\mathbb{C}[z]$-morphism $\tilde{\lambda}_{*}: \mathcal{L}\left(\nu_{i}\right) \rightarrow \mathcal{L}(X)_{X_{i}}$ with $\mathcal{L}_{\alpha}^{\circ}(X)_{X_{i}}=\tilde{\lambda}_{*}\left(\mathcal{L}\left(\nu_{i}\right)\right) \cap \mathcal{L}^{\circ}(X)$ showing that $\mathcal{L}_{\alpha}^{\circ}(X)_{X_{i}}$ is a $\mathbb{C}[z]$-semi-algebraic subset of $\mathcal{L}(X)$. Moreover we also have a $\mathbb{C}[z]$-morphism $\lambda_{*}: \mathcal{L}\left(\nu_{i} / \Gamma_{i}\right) \rightarrow \mathcal{L}(X)$ inducing a bijection $\lambda_{*}: \mathcal{L}\left(\nu_{i}\right) / \Gamma_{i} \simeq\left(\mathcal{L}\left(\nu_{i}\right) / \Gamma_{i}\right) \cap \lambda_{*}^{-1}\left(\mathcal{L}^{\circ}(X)\right)$. 
Proceeding as in 3.3[7] we get $\mu^{\text {orb }}\left(\mathcal{L}_{\alpha}^{\circ}(X)_{X_{i}}\right)=\mathbb{L}^{w(\alpha)} \mu_{\mathcal{L}\left(\nu_{i} / R_{i}\right)}^{\text {orb }}\left(\mathcal{L}\left(\nu_{i}\right) / R_{i}\right)$. But we have as in $3.4[7$ that

$$
\begin{aligned}
\mu_{\mathcal{L}\left(\nu_{i} / R_{i}\right)}^{\text {orb }}\left(\mathcal{L}\left(\nu_{i}\right) / R_{i}\right) & =\left[\pi_{r}\left(\mathcal{L}\left(\nu_{i}\right)\right) / R_{i}\right] \mathbb{L}^{-(r+1) \operatorname{rank}\left(\nu_{i}\right) / m}+R_{M}^{\prime} \\
& =\left[X_{i}^{\alpha}\right] \mathbb{L}^{(r+1) \operatorname{rank}\left(\nu_{i}\right) / m} \mathbb{L}^{-(r+1) \operatorname{rank}\left(\nu_{i}\right) / m}+R_{M}^{\prime}
\end{aligned}
$$

where $\lim _{M \rightarrow \infty} R_{M}^{\prime}=0$. This proves that

$$
\mu^{\text {orb }}\left(\mathcal{L}_{\alpha}^{\circ}(X)_{X_{i}}\right)=\left[X_{i}^{\alpha}\right] \mathbb{L}^{w(\alpha)},
$$

finishing the proof of theorem 1.4 .1

3.4.5. Finally by applying $\chi_{h}, \chi_{h n}$ and $\chi_{\text {top }}\left(\right.$ cf. $\S 2, \S 4[14)$ to $\mu^{\text {orb }}(\mathcal{L}(X))=$ $\mu^{\text {orb }}(\mathcal{L}(Y))$ we obtain theorem 1.3 .1

3.5. We would like to thank very enlightening conversations with R. Kulkarni, T. Nevins, M. Reid, Y. Ruan, G. Segal and B. Uribe during the preparation of this work. The second author would also like to thank M. Artin and D.A. Cox for useful email correspondences.

\section{REFERENCES}

1. Dan Abramovich, Tom Graber, and Angelo Vistoli, Algebraic orbifold quantum products, in: Orbifolds in Mathematics and Physics (Madison, WI, 2001), A. Adem et al., eds., Contemp. Math. 310, Amer. Math. Soc., Providence, RI, 2002, pp. 1-24.

2. Victor V. Batyrev, Stringy Hodge numbers of varieties with Gorenstein canonical singularities, Integrable systems and algebraic geometry (Kobe/Kyoto, 1997), World Sci. Publishing, River Edge, NJ, 1998, pp. 1-32.

3. - Non-Archimedian integrals and stringy Euler numbers of log terminal pairs, J. Eur. Math. Soc. (JEMS) 1 (1999), no. 1, 5-33.

4. W. Chen and Y. Ruan, A new cohomology theory of orbifold, arXiv:math.AG/0004129 (2000).

5. Alastair Craw, An introduction to motivic integration, arXiv:math.AG/9911179 (1999).

6. Jan Denef and François Loeser, Germs of arcs on singular algebraic varieties and motivic integration, Invent. Math. 135 (1999), no. 1, 201-232.

7. _ Motivic integration, quotient singularities and the McKay correspondence, Compositio Math. 131 (2002), no. 3, 267-290.

8. L. Dixon, J. Harvey, C. Vafa and E. Witten, Strings on orbifolds, I, II, Nucl. Phys., B 261 (1985), 678-686, B 274 (1986), 285-314.

9. A. Haefliger and Quach Ngoc Du, Appendice: une présentation du groupe fondamental d'une orbifold, Astérisque (1984), no. 116, 98-107, Transversal structure of foliations (Toulouse, 1982).

10. Y. Ito and M. Reid, The McKay correspondence for finite subgroups of $S L(3, C)$, in: Higher Dimensional Complex Varieties (Trento, Jun 1994), M. Andreatta et al., eds., de Gruyter, 1996, 221-240.

11. D. Kaledin, McKay correspondence for symplectic quotient singularities, Invent. Math. 148 (2002), no. 1, 151-175.

12. T. Kawasaki, The signature theorem for V-manifolds, Topology 17 (1978), 75-83.

13. M. Kontsevich, Lecture at Orsay, Thu 7th Dec 1995.

14. Eduard Looijenga, Motivic measures, Astérisque (2002), no. 276, 267-297, Séminaire Bourbaki, Vol. 1999/2000.

15. Domingo Luna, Slices étales, Sur les groupes algébriques, Soc. Math. France, Paris, 1973, pp. 81-105. Bull. Soc. Math. France, Paris, Mémoire 33.

16. E. Lupercio and B. Uribe, Loop groupoids, gerbes and twisted sectors on orbifolds, in: Orbifolds in Mathematics and Physics (Madison, WI, 2001), A. Adem et al., eds., Contemp. Math. 310, Amer. Math. Soc., Providence, RI, 2002, pp. 163-184.

17. _ Gerbes over orbifolds and K-theory, arXiv:math.AT/0105039 (2001). 
18. I. Moerdijk, Orbifolds as groupoids: an introduction.,in: Orbifolds in Mathematics and Physics (Madison, WI, 2001), A. Adem et al., eds., Contemp. Math. 310, Amer. Math. Soc., Providence, RI, 2002, pp. 205-222.

19. D. Pronk, Etendues and stacks as bicategories of fractions, Comm. Math. 102 (1996), 243303.

20. Miles Reid, La correspondance de McKay, Astérisque (2002), no. 276, 53-72, Séminaire Bourbaki, Vol. 1999/2000.

21. Y. Ruan, Stringy geometry and topology of orbifolds, in: Symposium in Honour of C.H. Clemens (Salt Lake City, UT, 2000), Contemp. Math. 312, Amer. Math. Soc., Providence, RI, 2002, pp. 187-233.

22. Burt Totaro, The resolution property for schemes and stacks, J. Reine Angew Math. (to appear), arXiv:math.AG/0207210 (2002).

23. Takehiko Yasuda, Twisted jet, motivic measure and orbifold cohomology, Compositio Math. (to appear), arXiv:math.AG/0110228 (2001).

Department of Mathematics, University of Wisconsin at Madison, Madison, Wi 53706

Department of Mathematics, Michigan State University, East Lansing, Mi 48824

E-mail address: lupercio@math.wisc.edu, poddar@math.msu.edu 